\title{
Ueber die Hydrolyse des Caseins durch Salzsäure.
}

Von

Emil Fischer.

(Aus dem I. chemischen Institut der Universität Berlin.)

(Der Redaction zugegangen am 5. Juli 1901.)

Unter den Spaltungsprodukten, welche das Casein mit Salzsäure liefert, hat man bisher mit Sicherheit festgestellt: Tyrosin und Leucin, Asparagin- und Glutaminsäure, Arginin und Lysin. Endlich wurde von Cohn ${ }^{1}$ ) noch eine Verbindung $\mathrm{C}_{12} \mathrm{H}_{22} \mathrm{~N}_{2} \mathrm{O}_{2}$ beobachtet, welche mit dem Leucinimid isomer ist und aller Wahrscheinlichkeit nach secundär aus einer Aminosäure gebildet wird.

Dass die erwähnten Produkte aber keineswegs die einzigen Bestandtheile des Caseins sind, zeigen alle Angaben über ihre Mengen, die um so kleiner werden, je mehr sich die Beobachter bemüht haben, reine Präparate darzustelle̊n. Die letżten Angaben dieser Art rühren von Cohn ${ }^{2}$ ) her, welcher eine sogenannte quantitative Spaltung des Caseins beschrieben hat. Mehr als die Hälfte seiner Produkte sind undefinirbare Sirupe, und auch die krystallisirten Partien, wie das rohe Leucin, tragen alle Kennzeichen von Gemischen. Es war deshalb von vornherein zu erwarten, dass eine vollkommenere Untersuchung der Hydrolyse noch eine Reihe von bisher übersehenen Stoffen zu Tage fördern werde.

Aber Angesichts der vielen Mühe und Sorgfalt, welche bei den älteren Arbeiten auf diesen Gegenstand verwandt wurde, konnte man sich der Ueberzeugung nicht verschliessen, dass mit den bisher gebrauchten Methoden neue Resultate nur sehr schwer zu erzielen seien.

1) Diese Zeitschrift, Bd. XXII, S. 170, 1897; Bd. XXIX, S. 283, 1900.

2) Diese Zeitschrift, Bd. XXVI, S. 395, 1899. 
Wie ich vor Kurzem mitgetheilt habe, ${ }^{1}$ ) ist es mir nun gelungen, für die Scheidung und Reinigung von Aminosäuren ein neues Verfahren aufzufinden, welches auf der fractionirten Destillation ihrer Ester beruht. Die Aminosäuren werden dabei zunächst nach der Angabe von Curtius²) mit Salzsäure und Alkohol verestert, dann folgt die Abscheidung aus den Hydrochloraten nach meiner Beobachtung durch concentrirtes Alkali bei niederer Temperatur, und schliesslich die Destillation der Ester unter sehr geringem Druck.

Ich habe mich zunächst darauf beschränkt, das Verfahren für die Monoaminsäuren auszubilden, und will nun beim Casein die grossen Vortheile desselben zeigen. Es ist dadurch gelungen, unter seinen Spaltungsprodukten folgende bisher übersehene Stoffe

$$
\begin{aligned}
& \text { Aminovaleriansäure, } \\
& \text { Phenylalanin, } \\
& \alpha \text {-Pyrrolidincarbonsäure }
\end{aligned}
$$

mit Sicherheit nachzuweisen und die Anwesenheit von Glycocoll sowie von anderen nicht näher charakterisirten Aminosäuren wahrscheinlich zu machen. ${ }^{3}$ )

Das meiste Interesse darunter verdient die $\alpha$-Pyrrolidincarbonsäure, welche bisher nur als synthetisches racemisches Produkt bekannt war, ${ }^{4}$ ) aber aus dem Casein auch als optisch active Form erhalten werden konnte. $\mathrm{Ob}$ dieselbe ebenso wie die anderen Aminosäuren als primäres Spaltungsprodukt $\mathrm{zu}$ betrachten ist, soll später discutirt werden.

Als Material für die Untersuchung, welche mit grösseren Mengen durchgeführt werden musste, diente die beste Sorte des käuflichen Caseins (puriss. Merck). Wie weit die Resultate auch für das nach der Vorschrift von Hammarsten dargestellte Präparat oder für das Pflanzencasein gelten, bleibt noch festzustellen.

1) Ber. d. deutsch. chem. Ges., Bd. 34, 433, 1901.

2) Journ. f. prakt. Ch., Bd. 37, 159, 1888.

3) Vgl. die vorläufige Anzeige, Berichte d. d. chem. Ges., Bd. 34, 447.

4) Willstätter, Berichte d. d. chem. Ges., Bd. 33, 1160 und E. Fischer, ebenda, Bd. 34, 454 (1901). 
Zersetzung des Caseins durch Salzsäure.

Die Hydrolyse, welche Hlasiwetz und Habermann ${ }^{1}$ ) durch langes Kochen mit verdünnter Säure unter Zusatz von Zinnchlorür bewerkstelligten, wird nach Cohn (l. c.) besser und rascher mit concentrirter Salzsäure ohne Zinnsalz ausgeführt.

Seiner Angabe entsprechend wurden 500. $\mathrm{g}$ Casein mit $1^{1}$ /2 l Salzsäure (1,19 sp. Gew.) übergossen und im Laufe von $1^{1 / 2}$ Stunden wiederholt durchgeschüttelt, bis der grösste Theil mit schmutzig violetter Farbe gelöst war. Dann wurde 6 Stunden am Rückflusskühler gekocht und die erkaltete Flüssigkeit von den ausgeschiedenen Fettsäuren durch Filtration getrennt.

Von hieran bin ich der Vorschrift Cohn's, welcher sofort die Salzsäure durch Abdampfen und Behandlung mit Kupferoxydul entfernt, nicht mehr gefolgt, sondern habe zunächst nach dem Vorgange von Hlasiwetz und Habermann die Glutaminsäure durch Krystallisation des Chlorhydrates abgeschieden.

$\mathrm{Zu}$ dem Zweck wurde die Flüssigkeit auf etwa $3 / 41$ eingedampft und in der Kälte mit gasförmiger Salzsäure gesättigt. Sie blieb dann 3 Tage im Eisschrank stehen, wobei die salzsaure Glutaminsäure als dicker Krystallbrei ausfiel. Um sie abfiltriren zu können, wurde mit dem gleichen Volumen eiskalten Alkohols vermischt und auf der Pumpe abgesaugt. Will man das Salz rein darstellen, so wird es in wenig Wasser gelöst, mit etwas Thierkohle gekocht und mit Chlorwasserstoff wieder abgeschieden. Nach einmaliger Wiederholung dieser Operation ist das Präparat nahezu farblos. Die Ausbeute betrug $50 \mathrm{~g}$, mithin 10\% des Caseins.

Veresterung der Aminosäuren.

Für diese Operation kann direkt das Filtrat von der Glutaminsäure dienen, nur ist es nöthig, das darin enthaltene Wasser möglichst vollständig $\mathrm{zu}$ entfernen. $\mathrm{Zu}$ dem Zweck wird, am besten unter vermindertem Druck, eingedampft, der

1) Liebig's Annalen, Bd. 169, 150, 1873. 
dunkelbraune, sirupdicke Rückstand mit $1^{1 / 2} 1$ absolutem Alkohol durchgerührt und mit gasförmiger Salzsäure, zuletzt unter Erwärmen auf dem Wasserbade, gesättigt. Da bei der Veresterung wieder erhebliche Mengen von Wasser entstehen, welche die Vollständigkeit der Reaction hindern, so ist es vortheilhaft, von Neuem unter vermindertem Druck einzudampfen, wieder in 11/2 l Alkohol zu lösen und abermals mit Salzsäure zu sättigen. Eine zweite Wiederholung der ganzen Operation steigert noch die Ausbeute an Ester. Jetzt wird die Flüssigkeit der bequemen Handhabung wegen in 4 Portionen getheilt und bei einer $40^{\circ}$ nicht übersteigenden Temperatur des Wasserbades unter stark vermindertem Druck zum dicken Sirup verdampft. Dieser enthält die Hydrochlorate der Aminoester; um letztere in Freiheit zu setzen, verfährt man folgendermassen:

Der dicke Sirup wird direkt in dem Destillationskolben ungefähr mit dem halben Volumen Wasser versetzt. Jetzt fügt man zu der sorgfältig mit einer Kältemischung gekühlten Flüssigkeit vorsichtig soviel starke Natronlauge, dass die freie Salzsäure ungefähr neutralisirt ist, und dann eine möglichst concentrirte Lösung von Kaliumcarbonat und ziemlich viel Aether. Diese erste Operation hat den Zweck, die schwächer basischen Ester der Asparagin- und Glutaminsäure, welche gegen freies Alkali besonders empfindlich sind, abzuscheiden. Nach gutem Durchschütteln wird der Aether abgegossen, durch neuen ersetzt, und zu der wiederum sehr sorgfältig gekühlten Masse in verschiedenen Portionen 33\% oige Natronlauge und festes Kaliumcarbonat zugegeben. Nach jedesmaligem Zusatz wird kräftig umgeschüttelt, um das Alkali in der steifen Masse zu vertheilen und den frei gewordenen Ester sofort in die ätherische Lösung überzuführen.

Es ist vortheilhaft, den Aether mehrmals zu erneuern. Die Menge des Alkalis muss wenigstens so gross sein, dass sie zur Bindung sämmtlicher Salzsäure ausreicht, und Kaliumcarbonat ist soviel zuzufügen, dass die Salzmasse einen dicken Brei bildet; denn nur dann werden die in Wasser äusserst leicht löslichen Ester der einfachen Aminosäuren völlig aus- 
gesalzen. Ganz besonders gilt das für die Fälle, wo Glycocoll und Alanin zu isoliren sind.

Die vereinigten ätherischen Auszüge, welche braun gefärbt sind, werden etwa 5 Minuten mit Kaliumcarbonat geschüttelt, dann abgegossen und 12 Stunden mit entwässertem Natriumsulfat getrocknet, da die übrigen Trockenmittel, wie Aetzkali, Calcium- und Baryumoxyd oder selbst das Kaliumcarbonat, bei längerer Einwirkung etwas Ester zersetzen. Nach dem Verdampfen des Aethers wird der Rückstand bei 8-15 mm Druck über freier Flamme destillirt.

Bis $40^{\circ}$ geht in der Regel noch etwas Alkohol über, dann wurden bei einem Versuch, der mit $500 \mathrm{~g}$ Casein recht sorgfältig durchgeführt war, folgende Fractionen erhalten:

$$
\begin{aligned}
& 40-55^{\circ} \text { (bei } 10 \mathrm{~mm} \text { ) } 7 \mathrm{~g} \\
& 55-80^{\circ}(\triangleright 9>) 13 \text {, } \\
& 80-100^{\circ} \text { ( } 9 \text {, ) } 97 \text {. } \\
& 100-130^{\circ} \text { ( } 9 \text {, ) } 27 \text {, } \\
& 130-160^{\circ}\left(\Rightarrow 9>\frac{29 \text {. }}{173 \mathrm{~g} .}\right.
\end{aligned}
$$

Längeres Kochen des Caseins mit Salzsäure ändert an dem Resultat nur wenig. Bei einem Versuche, wo wiederum 500 g Casein 36 Stunden mit der Säure gekocht waren, betrug die Gesammtmenge der in Aether löslichen Produkte $280 \mathrm{~g}$ und der bis $170^{\circ}$ bei $16 \mathrm{~mm}$. destillirende Ester $200 \mathrm{~g}$, wobei aber die letzte Fraction schon erhebliche Mengen von Zersetzungsprodukten enthielt. Die Produkte sind ebenfalls die gleichen wie beim kürzeren Kochen.

Zur weiteren Trennung der Ester wurden mehrere der obigen Fractionen nochmals einer zweimaligen systematischen Fractionirung unterworfen und bei $10 \mathrm{~mm}$. Druck folgende 8 Präparate aus $1 \mathrm{~kg}$ Casein gewonnen:
1. $40-55^{\circ} 14 \mathrm{~g}$
2. $55-65^{\circ} 14$,
3. $65-80^{\circ} 25$,
4. $80-85^{\circ} 165$,
5. $85-110^{\circ} 18$,
6. $110-120^{\circ} 40$,
7. $120-130^{\circ} 28$,
8. $130-160^{\circ} \frac{8 \mathrm{~g}}{312 \mathrm{~g}}$ 
Sämmtliche Fractionen sind noch Gemische, die durch weitere Destillation nicht gereinigt werden können. Zur Scheidung in die einzelnen Bestandtheile wurden deshalb die Ester in die Aminosäuren zurückverwandelt.

Diese Verseifung lässt sich bei den bis $85^{\circ}$ siedenden Produkten, welche nur aus Estern der Monaminosäuren bestehen, am bequemsten durch mehrstündiges Kochen mit Wasser am Rückflusskühler bewerkstelligen. Bei den höher siedenden Teilen, welche Asparagin- und Glutaminsäure enthalten, ist die Verseifung durch Erwärmen mit Barytwasser auszuführen.

$\mathrm{Zu}$ beachten ist, dass die Ester sich beim Aufbewahren allmählich zersetzen, und zwar um so rascher, je kleiner das Molekül ist. Es empfiehlt sich deshalb, die Verseifung möglichst bald und zwar bei den niedrigsten Fractionen schon innerhalb 24 Stunden vorzunehmen.

\section{Fraction $40-55^{\circ}$.}

Wie die Rückverwandlung in die Aminosäuren zeigte, bestand diese Fraction noch etwa zu zwei Drittheilen aus Alkohol, dessen völlige Entfernung beim Eindampfen der salzsauren Ester nicht möglich ist.

Beim längeren Stehen schied die Flüssigkeit eine amorphe feste Masse ab, welche eine sehr schöne Biuretreaction gab. Wie ich früher dargelegt habe, ${ }^{1}$ ) wird dadurch die Anwesenheit von Glycocollester sehr wahrscheinlich gemácht. Um denselben als Hydrochlorat zu isoliren, wurde eine Probe der Flüssigkeit in der doppelten Menge absoluten Alkohols gelöst, mit gasförmiger Salzsäure gesättigt, in einer Kältemischung gekühlt und mit einem winzigen Kryställchen von salzsaurem Glycocollester geimpft. Nach 2 Stunden war in der That eine ziemlich erhebliche Krystallisation eingetreten und das Produkt zeigte nach dem Lösen in Alkohol und Fällen mit Aether sowohl den äusseren Habitus wie den Schmelzpunkt (gef. $145^{\circ}$ corr.) des salzsauren Glycocollesters. Da aber die Menge im Ganzen recht gering war, so halte ich es für wahrscheinlich, dass

1) Ber. d. deutsch. chem. Ges., Bd. 34, 442 (1901). 
dieses Glycocoll von einer Verunreinigung des Caseins herrührt.

Der grösste Teil der Fraction wurde zur Verseifung der Ester mit der fünffachen Menge Wasser 5 Stunden am Rückflusskühler gekocht und die Lösung concentrirt, bis in der Kälte Krystallisation erfolgte. Die erste Krystallisation (0,34 g) gab bei der Analyse Zahlen, welche sich am meisten den für Aminovaleriansäure verlangten Werthen nähern.

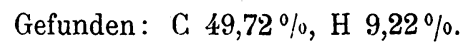

Berechnet: $\approx 51,29 \%, \otimes 9,40 \%$.

Die Mutterlauge hinterliess beim vollständigen Verdampfen noch 2,5 g Aminosäure. Sie wurde in wenig heissem Wasser gelöst, die in der Kälte ausfallenden Blättchen durch Filtration entfernt, und die Mutterlauge in $400 \mathrm{ccm}$. heissen Alkohol (95\%) eingegossen. Die Menge der nach längerem Stehen in der Kälte abgeschiedenen Krystalle betrug 1,2 g. Sie wurden für die Analyse bei $110^{\circ}$ getrocknet. Die Zahlen passen recht gut auf Aminopropionsäure.

$0,2185 \mathrm{~g}$ Substanz gaben $29,4 \mathrm{ccm}$. N $\left(21^{\circ}, 767,5 \mathrm{~mm}\right.$.).

0,1850 » > $>0,2725 \mathrm{~g} \mathrm{CO}_{2}$ und $0,1310 \mathrm{~g} \mathrm{H}_{2} \mathrm{O}$.

$\mathrm{C}_{8} \mathrm{H}_{7} \mathrm{O}_{2} \mathrm{~N}$. Berechnet: $40,45 \% \mathrm{C}, 7,86 \% \mathrm{H}, 15,73 \% \mathrm{~N}$. Gefunden: $40,17 \% \gg 7,87 \%, 15,50 \%$,

Das Hydrochlorat drehte nach rechts.

Leider reichte die Menge des Präparates nicht aus, um durch Darstellung von Derivaten die Identität mit dem Alanin festzustellen. Ich halte deshalb die Wiederholung des Versuches mit grösseren Mengen für nöthig.

\section{Fraction $55-65^{\circ}$.}

Diese Fraction enthält Aminovaleriansäure und zwar, wie es scheint, als Hauptbestandtheil. Der Ester wurde durch Kochen mit Wasser verseift und die Aminosäuren zunächst ins Kupfersalz verwandelt. Nachdem der schwerer lösliche Theil des Salzes, welcher übrigens den Metallgehalt des aminovaleriansauren Kupfers schon besass (gefunden: $\mathrm{Cu} 21,51 \%$, berechnet: $21,49 \%$ ), entfernt war, wurde die Aminosäure regenerirt und zweimal aus Wasser umkrystallisirt. Die so 
erhaltenen farblosen glänzenden Blättchen gaben nach dem Trocknen bei $100^{\circ}$ Zahlen, welche ziemlich gut auf Aminovaleriansäure stimmen. Leider reichte in Folge eines grossen Verlustes die Menge für eine ausführliche Untersuchung nicht aus, aber ich zweifle nicht daran, dass das Produkt mit der sogleich näher $\mathrm{zu}$ beschreibenden Aminovaleriansäure identisch war.

$0,2000 \mathrm{~g}$ Substanz gaben 0,3689 $\mathrm{g} \mathrm{CO}_{2}$ und $0,1673 \mathrm{~g} \mathrm{H}_{2} \mathrm{O}$.

$\mathrm{C}_{5} \mathrm{H}_{11} \mathrm{O}_{2} \mathrm{~N}$. Berechnet: $51,28 \% \mathrm{C}, 9,40 \% \mathrm{H}$.

Gefunden: $51,47 \%$, $9,39 \%$ 》

\section{Fraction $65-80^{\circ}$.}

Neben kleinen Mengen von Leucin und Pyrrolidincarbonsäure findet sich auch hier Aminovaleriansäure, aber ihre Isolirung und Reinigung ist so schwierig, dass es nicht möglich war, genügendes Material zu beschaffen, um ihre Constitution aufzuklären.

Zunächst wurde das Gemisch der Ester durch Kochen mit der fünffachen Menge Wasser in der gleichen Weise wie die Fraction $80-85^{\circ}$ verseift. Das Gewicht der Aminosäuren betrug $12 \mathrm{~g}$. $10 \mathrm{~g}$ wurden in 21 Wasser gelöst und 1 Stunde mit überschüssigem gefällten Kupferoxyd gekocht. Das Filtrat schied beim Eindampfen ziemlich bald ein schwer lösliches Kupfersalz ab, welches mehrmals abgehoben und mit wenig Wasser ausgekocht wurde, wobei die Laugen zur ursprünglichen Lösung zurückgegeben wurden. Die Menge des schwer löslichen Kupfersalzes betrug 2,1 g und die Bestimmung des Kupfergehaltes deutete auf ein Gemisch von etwa gleichen Theilen Leucin und Aminovaleriansäure. Das Filtrat hinterliess beim Verdampfen die leicht löslichen Kupfersalze. Beim Auskochen mit absolutem Alkohol gingen davon 0,9 $\mathrm{g}$ in Lösung, welche wohl im Wesentlichen l-pyrrolidincarbonsaures Kupfer waren.

Der in Alkohol unlösliche Theil wurde dann mehrmals mit ungefähr $100 \mathrm{ccm}$. Wasser bei $70-80^{\circ}$ ausgelaugt, wobei wieder $0,8 \mathrm{~g}$ schwer lösliches Kupfersalz zurückblieb. Die wässerige Lösung wurde mit Schwefelwasserstoff vom Kupfer 
befreit, zur Trockne verdampft und der Rückstand mit $25 \mathrm{ccm}$. absolutem Alkohol ausgekocht. Dabei ging die racemische Pyrrolidincarbonsäure in Lösung, ihre Menge betrug 1,5 g.

Der in Alkohol unlösliche Theil wurde in Wasser gelöst, mit etwas Thierkohle gekocht und das eingeengte Filtrat mit dem gleichen Volumen Alkohol versetzt. Dabei schieden sich 2,5 g Aminosäure ab, welche folgende Zahlen gab:

$0,1885 \mathrm{~g}$ Substanz lieferten $0,3444 \mathrm{~g} \mathrm{CO}_{2}$ und $0,1561 \mathrm{~g} \mathrm{H}_{\mathbf{2}} \mathrm{O}$.

$\mathrm{C}_{5} \mathrm{H}_{11} \mathrm{O}_{2} \mathrm{~N}$. Berechnet: $51,28 \% \mathrm{C}, 9,40 \% \mathrm{H}$.

Gefunden: $49,83 \%$, $9,20 \%$,

Man ersieht daraus, dass das Präparat noch keineswegs reine Aminovaleriansäure war. In 20\% iger Salzsäure gelöst gab es $[\alpha]_{D}^{20}=+27,1^{\circ}$. Zur weiteren Reinigung wurde die Substanz in $20 \mathrm{ccm}$. kochendem Wasser gelöst und mit $40 \mathrm{ccm}$. Alkohol wieder gefällt. Die so erhaltenen schönen glänzenden Blättchen schmolzen in geschlossenem Capillarrohr unter Gasentwicklung bei 292-295 (corr.). Nach der Ahalyse war auch dieses Präparat keine ganz reine Aminovaleriansäure.

$0,2110 \mathrm{~g}$ Substanz gaben $0,3891 \mathrm{~g} \mathrm{CO}_{2}$ und $0,1728 \mathrm{~g} \mathrm{H}_{2} \mathrm{O}$. Gefunden: $50,30 \% \mathrm{C}, 9,10 \% \mathrm{H}$.

Zur weiteren Charakterisirung wurde deshalb die Aminosäure zuerst racemisirt und dann mit Phenylcyanat gekuppelt. Das Erstere geschah durch 24 stündiges Erhitzen mit der dreifachen Menge Barythydrat und der zwanzigfachen Menge Wasser auf $175^{\circ}$. Die in der üblichen Weise abgeschiedene Aminosäure bildete glänzende Blättchen, welche sich von dem r-Leucin durch ihre viel grössere Löslichkeit in kaltem Wasser unterschieden.

$1 \mathrm{~g}$ von dem Präparate wurde in $10 \mathrm{ccm}$. Normalnatronlauge gelöst und bei $0^{\circ}$ unter kräftigem Schütteln tropfenweise mit Phenylcyanat solange versetzt, bis eine Abscheidung von Diphenylharnstoff stattfand.

Aus dem alkalischen Filtrat fiel beim Ansäuern die Phenylcyanatverbindung als zähes Harz aus, welches aber bald krystallinisch erstarrte.

Aus heissem 50\%igen Alkohol krystallisirte das Produkt in glänzenden, oft sechsseitigen Blättchen, welche bei $157-158^{\circ}$ unter Zersetzung schmolzen. 
Schöner als die Phenylcyanatverbindungen selbst sind in der Regel ihre Anhydride, das trifft auch im vorliegenden Falle zu. Zur Bereitung des Anhydrids wurde in der bekannten Weise die Verbindung in der hundertfachen Menge 20\% iger heisser Salzsäure gelöst, eine halbe Stunde gekocht und dann die Flüssigkeit auf etwa 1/3 Volumen eingedampft. Beim Erkalten schieden sich farblose, zu Büscheln gruppirte Nadeln ab, welche zur Analyse nochmals aus siedendem Wasser umkrystallisirt und im Vacuum über Schwefelsäure getrocknet wurden.

$0,1838 \mathrm{~g}$ Substanz gaben $0,445 \breve{6} \mathrm{~g} \mathrm{CO}_{2}$ und $0,1100 \mathrm{~g} \mathrm{H}_{2} \mathrm{O}$.

$\begin{array}{lll}0,1777 \gg & \gg, 7 \mathrm{ccm} . \mathrm{N}(22 \%, 767 \mathrm{~mm} .) . \\ \mathrm{C}_{12} \mathrm{H}_{14} \mathrm{O}_{2} \mathrm{~N}_{2} . & \text { Berechnet: } 66,05 \% \mathrm{C}, 6,42 \% \mathrm{H}, 12,84 \% \mathrm{~N} . \\ & \text { Gefunden: } 66,12 \% \gg 6,62 \% \gg 12,72 \% \text { }\end{array}$

- Die erhaltenen Zahlen stimmen recht gut auf die Formel $\mathrm{C}_{12} \mathrm{H}_{14} \mathrm{~N}_{2} \mathrm{O}_{2}$. Das würde dem Phenylhydantoinderivat einer $\alpha$-Aminovaleriansäure von folgender Structur

$$
\left.\right|_{\mathrm{CO}}-\mathrm{CH}-\mathrm{CH}_{2}-\mathrm{CH}_{2}-\mathrm{CH}_{3}
$$

oder einer isomeren Verbindung entsprechen.

Das Präparat schmolz auch nach mehrmaligem Umkrystallisiren bei $117^{\circ}$ (corr.), aber vielfältige Erfahrung hat mich gelehrt, dass Constanz des Schmelzpunktes bei solchen Derivaten der Aminosäuren keine Garantie für die Reinheit ist, und so kann ich auch im vorliegenden Falle für die Einheitlichkeit des Präparates nicht einstehen, zweifle aber nicht daran, dass es der Hauptmenge nach das Derivat einer Aminovaleriansäure ist.

Untersuchung der Fraction $80-85^{\circ}$.

Das Produkt ist in kaltem Wasser leicht löslich, scheidet sich aber beim Erwärmen der concentrirten Lösung zum Theil wieder ölig ab. Seine specifische Drehung war $+5,8^{\circ}$.

$80 \mathrm{~g}$ wurden mit der 10 fachen Menge Wasser 6-7 Stunden gekocht, bis das Oel gänzlich und die alkalische Reaction der Flüssigkeit nahezu verschwunden war. Durch successives Eindampfen und Erkaltenlassen der Lösung resultirten $3 \mathrm{Kry-}$ 
stallisationen von 14, 9 und 8 g. Eine 4. Krystallisation wurde dann aus dem Filtrat durch Zusatz der gleichen Menge Alkohol gewonnen. Die Mutterlaugen dienten, wie später beschrieben wird, zur Isolirung der beiden isomeren Pyrrolidincarbonsäuren.

Die 4 Krystallisationen bestehen zum allergrössten Theile aus Aminocapronsäuren und enthalten wahrscheinlich eine kleine Menge von Aminovaleriansäure. Um diese abzutrennen, wurden durch Umkrystallisiren 7 Fractionen hergestellt, welche auf ihr optisches Drehungsvermögen in 20\%iger Salzsäure untersucht sind.
A. $4,7 \mathrm{~g}[\alpha]_{\mathrm{D}}^{200}=+12,0^{\circ}$
B. $6, \tilde{0} \gg,=+22,4^{0}$
C. $3,7 \diamond \diamond=+21,7^{\circ}$
D. $6,7 \gg,=+23,1^{\circ}$
E. $7,0 \curvearrowright,=+27,3^{\circ}$
F. $6,5, \gg=+29,0^{\circ}$
G. $\frac{6,0 \text { » }}{41,1 \mathrm{~g} .}$

Die Fractionen A und D wurden analysirt und zeigten die Zusammensetzung der Aminocapronsäure. Dass sie aber ebensowenig wie die zwischenliegenden Theile B und C einheitliche Präparate sind, zeigt das Schwanken des Drehungsvermögens. Unzweifelhaft bestehen sie zum grössten Theil aus dem gewöhnlichen Leucin, welches in der ersten Fraction zum Theil als racemische Form vorhanden ist. Für das active Leucin wird nun allerdings eine specifische Drehung von $+17,5^{\circ}$ von Schulze angegeben, und solange dieser Werth nicht als $\mathrm{zu}$ niedrig erkannt ist, muss man annehmen, dass in der Fraction D und höchst wahrscheinlich auch in B und $\mathrm{C}$ eine gleich zusammengesetzte, aber stärker drehende Aminosäure enthalten ist. Ihre Isolirung mit Hülfe des Kupfersalzes oder der Phenylcyanatverbindung habe ich vergeblich versucht.

Die Fractionen $F$ und $G$ sind Gemische von Leucin und Aminovaleriansäure. Man hätte erwarten sollen, dass diese beiden Säuren sich durch die Kupfersalze trennen liessen, da deren Löslichkeit in Wasser recht verschieden ist. Leider 
haben aber beide Salze die Neigung, żusammen zu krystallisiren. Man erhält gerade aus diesem Gemisch häufig prachtvoll dunkelblaue grosse blätterige Krystalle, die in heissem Wasser viel leichter löslich sind als Leucinkupfer. Ihre Analyse stimmt ungefähr auf ein Gemisch von gleichen Molekülen Leucinkupfer und aminovaleriansaurem Kupfer nebst einem Molekül Wasser:

$0,4372 \mathrm{~g}$ lufttrockenes Salz verloren bei $110^{\circ} 0,0244 \mathrm{~g}$ Wasser.

$$
\begin{array}{ll}
\mathrm{C}_{11} \mathrm{H}_{22} \mathrm{O}_{4} \mathrm{~N}_{2} \mathrm{Cu}+\mathrm{H}_{2} \mathrm{O} . & \begin{array}{l}
\text { Berechnet: } 5,50 \% \\
\text { Gefunden: } 5,58 \%
\end{array}
\end{array}
$$

$0,1984 \mathrm{~g}$ wasserfreies Salz gaben $0,3066 \mathrm{~g} \mathrm{CO}_{2}$ und $0,1276 \mathrm{~g} \mathrm{H}_{2} \mathrm{O}$.

$$
\begin{aligned}
& 0,1960 \text { » » } \gg 15,3 \mathrm{ccm} . \mathrm{N}\left(17^{\circ}, 750 \mathrm{~mm} .\right) \text {. }
\end{aligned}
$$

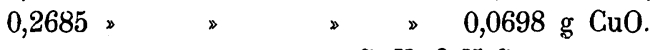

$$
\begin{aligned}
& \mathrm{C}_{11} \mathrm{H}_{22} \mathrm{O}_{4} \mathrm{~N}_{2} \mathrm{Cu} \text { : }
\end{aligned}
$$

Berechnet: $42,58 \% \mathrm{C}, 7,10 \% \mathrm{H}, 9,0 \% \mathrm{~N}, 20,48 \% \mathrm{Cu}$.

Gefunden: $42,15 \% \gg 7,14 \%$, $8,99 \% \gg 20,77 \%$,

Ob hier eine wirkliche molekulare Verbindung oder nur Mischkrystalle vorliegen, habe ich nicht weiter geprüft.

Die Isolirung der Aminovaleriansäure wurde hier besonders durch den Umstand erschwert, dass neben activen Säuren stets kleinere und wechselnde Mengen der Racemkörper vorhanden sind. In solchen Fällen ist es nach meiner Erfahrung rathsam, durch ein- bis zweitägiges Erhitzen mit überschüssigem Barytwasser auf $160-170^{\circ}$ im Autoclaven, wobei Glasgefässe $\mathrm{zu}$ vermeiden sind, völlig $\mathrm{zu}$ racemisiren, und dann erst die Trennung der einzelnen Aminosäuren durch Krystallisation oder durch die Kupfersalze vorzunehmen.

Ich habe diese Operation, speciell zur Isolirung der Aminovaleriansäure, mit dem rohen Gemisch der Aminosäuren, welches aus dem Ester der Fraction 80-850 entsteht, vorgenommen. Das racemisirte Produkt wurde dann durch Krystallisation aus Wasser in 3 Fractionen getheilt.

Die leicht lösliche Fraction wurde zur Entfernung der Pyrrolidincarbonsäure mehrmals mit absolutem Alkohol ausgekocht, der Rückstand in wenig Wasser gelöst und mit Alkohol gefällt. Das so erhaltene Produkt, dessen Menge ungefähr $5 \%$ des ursprünglichen Esters betrug, diente zur Darstellung der Phenylcyanatverbindung bezw. dessen Anhydrid. 
Die Analyse des letzteren gab Zahlen, welche auf das Derivat der Aminovaleriansäure stimmen.

0,1772 g Substanz gaben $0,4282 \mathrm{~g} \mathrm{CO}_{2}$ und $0,1065 \mathrm{~g} \mathrm{H}_{\mathbf{2}} \mathrm{O}$.

0,1085 > > > $12,4 \mathrm{ccm} . \mathrm{N}\left(24^{\circ}, 763 \mathrm{~mm}\right.$.).

$\mathrm{C}_{12} \mathrm{H}_{14} \mathrm{O}_{2} \mathrm{~N}_{2}$. Berechnet: $66,06 \% \mathrm{C}, 6,42 \% \mathrm{H}, 12,85 \% \mathrm{~N}$. Gefunden: $65,90 \%>6,68 \%>12,97 \%$,

Aber der Schmelzpunkt des Produktes $109^{\circ}$ lag $8^{\circ}$ niedriger als bei dem entsprechenden Präparat, welches aus der Fraction $65-80^{\circ}$ gewonnen war.

Die oben erwähnten wässerig-alkoholischen Mutterlaugen, welche neben gewöhnlichen Aminosäuren die beiden Pyrrolidincarbonsäuren enthalten, wurden nach Wegdampfen des Alkohols etwa auf $1 / 2$ Liter verdünnt und mit überschüssigem gefällten Kupferoxyd 11/2 Stunden gekocht. Beim Verdampfen des Filtrats blieben $28 \mathrm{~g}$ Kupfersalz zurück. Sie wurden fein gepulvert und $2 \mathrm{Mal}$ mit je $100 \mathrm{ccm}$. heissem absoluten Alkohol ausgelaugt. Ungelöst blieben $10 \mathrm{~g}$, und aus dem Filtrat fielen beim Erkalten noch $2 \mathrm{~g}$ aus, welche dann in Alkohol sehr schwer löslich waren.

Das unlösliche Kupfersalz enthält hauptsächlich die racemische $\alpha$-Pyrrolidincarbonsäure. Es wurde mit $300^{\circ} \mathrm{ccm}$. Wasser ausgekocht, von einer kleinen Menge Rückstand filtrirt, mit Schwefelwasserstoff zerlegt und das Filtrat im Vacuum zur Trockne verdampft. Beim Auslaugen des Rückstandes mit $40 \mathrm{ccm}$. heissem absoluten Alkohol, welcher die Pyrrolidincarbonsäure leicht löst, blieben 1,8 g Aminosäure zurück, welche nach einmaligem Umkrystallisiren aus Wasser analysirt wurden:

$0,1993 \mathrm{~g}$ Substanz gaben $0,3838 \mathrm{~g} \mathrm{CO}_{2}$ und $0,1717 \mathrm{~g} \mathrm{H}_{2} \mathrm{O}$.

$\mathrm{C}_{5} \mathrm{H}_{11} \mathrm{O}_{2} \mathrm{~N}$. Berechnet: $51,28 \% \mathrm{C}, 9,40 \% \mathrm{H}$.

$\mathrm{C}_{6} \mathrm{H}_{18} \mathrm{O}_{2} \mathrm{~N}$. $54,96 \%>9,92 \%$ >

Gefunden: $52,52 \%$, $9,58 \%$,

Die Zahlen deuten auf ein Gemisch von etwa \%/3 Aminovaleriansäure und 1/3 Aminocapronsäure.

Als bei einem späteren Versuch die entsprechende Substanz einmal aus Wasser und dann aus Wasser unter Zusatz von Alkohol umkrystallisirt wurde, ergab die Analyse folgende Zahlen: 
$0,1885 \mathrm{~g}$ Substanz gaben $0,3559 \mathrm{~g} \mathrm{CO}_{2}$ und $0,1603 \mathrm{~g} \mathrm{H}_{2} \mathrm{O}$. 0,1670 > \17,0 ccm. N (21,50, 761,5 mm.).

$\mathrm{C}_{5} \mathrm{H}_{11} \mathrm{O}_{2} \mathrm{~N}$. Berechnet: $51,28 \% \mathrm{C}, 9,40 \% \mathrm{H}, 11,95 \% \mathrm{~N}$. Gefunden: $51,49 \%>9,45 \%>11,69 \%$,

Die in der alkoholischen Lösung befindliche Pyrrolidincarbonsäure war nunmehr so gut wie frei von gewöhnlichen Aminosäuren. Sie wurde nach dem Verdampfen des Alkohols ins Kupfersalz zurückverwandelt und dies in 2 Fractionen $(3,8+1,4 \mathrm{~g})$ zerlegt. Die erste Fraction diente für die Analyse.

$1,2576 \mathrm{~g}$ tufttrockenes Salz verloren bei $108^{\circ} 0,1381 \mathrm{~g}$. 0,2210 > Substanz gaben $0,3325 \mathrm{~g} \mathrm{CO}_{2}$ und $0,1107 \mathrm{~g} \mathrm{H}_{8} \mathrm{O}$.

$$
\begin{array}{r}
\mathrm{C}_{10} \mathrm{H}_{16} \mathrm{O}_{4} \mathrm{~N}_{2} \mathrm{Cu}+2 \mathrm{H}_{2} \mathrm{O} \text {. Berechnet: } 10,99 \% \mathrm{H}_{2} \mathrm{O} . \\
\text { Gefunden: } 10,97 \% \text {, } \\
\mathrm{C}_{10} \mathrm{H}_{16} \mathrm{O}_{4} \mathrm{~N}_{2} \mathrm{Cu} \text {. Berechnet: } 41,16 \% \mathrm{C}, 5,49 \% \mathrm{H} . \\
\text { Gefunden: } 41,03 \% \triangleright 5,56 \%
\end{array}
$$

Die aus dem Salz in Freiheit gesetzte Säure gab nach einmaligem Umkrystallisiren und Trocknen bei $40^{\circ} \mathrm{im}$ Vacuum folgende Zahlen:

$0,1939 \mathrm{~g}$ Substanz gaben $0,3706 \mathrm{~g} \mathrm{CO}_{2}$ und $1307 \mathrm{~g} \mathrm{H}_{2} \mathrm{O}$.

$\mathrm{C}_{5} \mathrm{H}_{8} \mathrm{O}_{2} \mathrm{~N}$. Berechnet: $52,18 \% \mathrm{C}, 7,83 \% \mathrm{H}$.

Gefunden: $52,12 \%>7,88 \%$,

Die Substanz zeigte ganz das Verhalten der gewöhnlichen Pyrrolidincarbonsäure, wie es von Willstätter ${ }^{1}$ ) und dann von mir ${ }^{2}$ ) an den synthetischen Produkten gefunden wurde.

1-Pyrrolidincarbonsäure.

Ihr Kupfersalz ist in dem oben erwähnten alkoholischen Auszug enthalten und bleibt beim Verdampfen desselben als tiefblaue amorphe Masse zurück.

Es wurde in Wasser gelöst, mit Schwefelwasserstoff zerlegt, das Filtrat unter vermindertem Druck verdampft und der Rückstand zweimal mit absolutem Alkohol, im Ganzen $100 \mathrm{ccm}$, ausgekocht. Hierbei blieben $1,2 \mathrm{~g}$ gewöhnliche Aminosäure zurück, welche von der Pyrrolidincarbonsäure durch den viel höheren Schmelz- bezw. Zersetzungspunkt leicht zu unterscheiden ist. Das Rohprodukt enthielt $0,5 \% \mathrm{G}$ mehr, als für

1) Ber. d. deutsch. chem: Gesellsch., Bd. 33, 1160 (1900).

2) Ber. d. deutsch. chem. Gesellsch., Bd. 34, 458 (1901). 
Aminovaleriansäure verlangt wird. Nach einmaligem Umkrystallisiren aus Wasser, darauf aus Alkohol, gab die bei $125^{\circ}$ getrocknete Substanz folgende Zahlen:

$0,2000 \mathrm{~g}$ Substanz lieferten $0,3763 \mathrm{~g} \mathrm{CO}_{2}$ und $0,1677 \mathrm{~g} \mathrm{H}_{2} \mathrm{O}$.

0,1668 » $\gg 17,4 \mathrm{ccm} . \mathrm{N}\left(20,5^{\circ}, 754 \mathrm{~mm}\right.$.).

$\mathrm{C}_{5} \mathrm{H}_{11} \mathrm{O}_{2} \mathrm{~N}$. Berechnet: $51,28 \% \mathrm{C}, 9,40 \% \mathrm{H}, 11,95 \% \mathrm{~N}$. Gefunden: $51,31 \% \gg 9,32 \% \gg 11,95 \%$,

Die Lösung, welche in 4,7782 g Salzsäure (20\%) 0,2673 g Aminosäure, mithin 5,594\% enthielt und ein specifisches Gewicht von 1,10 besass, drehte im Decimeterrohr bei $20^{\circ}$ Natriumlicht $1,72^{\circ}$ nach rechts.

$$
[a]_{\mathrm{D}}^{20^{\circ}}=+27,95^{\circ}
$$

In geschlossener Capillare schmolz die Substanz unter starker Gasentwicklung bei $285-286^{\circ}$ (292-293 ${ }^{\circ}$ corr.).

Das alkoholische Filtrat von der Aminovaleriansäure hinterliess beim raschen Verdampfen die active Pyrrolidincarbonsäure als schwach gelbgefärbe krystallinische Masse. Die Ausbeute betrug $8,5 \mathrm{~g}$.

Zur weiteren Reinigung wurde die Säure entweder in heissem Alkohol gelöst und nach Zusatz von Aether der Krystallisation überlassen oder in wenig Wasser gelöst, nach Zusatz von Pyridin mit wenig Thierkohle gekocht und das Filtrat noch etwas eingeengt. Im letzteren Falle schieden sich hübsche flache Nadeln ab, welche aber beim späteren Trocknen an der Luft verwitterten. Schliesslich wurde das zuletzt genannte Präparat noch einmal aus Alkohol durch Eindunsten umkrystallisirt. Bei der Analyse gaben die drei Präparate folgende Werthe:

\section{Getrocknet bei $110^{\circ}$ :}

$0,1777 \mathrm{~g}$ Substanz lieferten $18,2 \mathrm{ccm} . \mathrm{N}\left(18^{\circ}, 765 \mathrm{~mm}\right.$.).

$0,1985 » \gg \quad 0,3713 \mathrm{~g} \mathrm{CO}_{2}$ und $0,1415 \mathrm{~g} \mathrm{H}_{2} \mathrm{O}$.
Gefunden: $51,01 \% \mathrm{C}, 7,92 \% \mathrm{H}, 11,91 \% \mathrm{~N}$.

II. Getrocknet im Vacuum über Schwefelsäure :

$0,2022 \mathrm{~g}$ Substanz lieferten $0,3795 \mathrm{~g} \mathrm{CO}_{2}$ und $0,1437 \mathrm{~g} \mathrm{H}_{2} \mathrm{O}$.

0,1719 > > > 0,3224 > > 0,1210 » ,

Gefunden: $51,19 \% \mathrm{C}, 7,89 \% \mathrm{H}$.

$$
51,15 \% \text { × } 7,89 \% \text { }
$$


III. Getrocknet im Vacuum bei $40^{\circ}$ :

$0,1950 \mathrm{~g}$ Substanz lieferten 0,3705 $\mathrm{g} \mathrm{CO}_{2}$ und 0,1403 $\mathrm{g} \mathrm{H}_{2} \mathrm{O}$.

Gefunden: $51,82 \% \mathrm{C}, 7,99 \% \mathrm{H}$

$\mathrm{C}_{5} \mathrm{H}_{8} \mathrm{O}_{\mathbf{8}} \mathrm{N}$. Berechnet: $52,18 \%>7,83 \%>12,17 \% \mathrm{~N}$.

In Folge der schwierigen Reinigung haben auch die optischen Bestimmungen schwankende Werthe ergeben, und ich halte es für möglich, dass selbst die höchste gefundene Zahl noch etwas zu niedrig ist.

I. Getrocknet über Phosphorsäureanhydrid im Vacuum. $4,5681 \mathrm{~g}$ wässerige Lösung, welche $0,4016 \mathrm{~g}$ Substanz, mithin $8,792 \%$ enthielt und das specifische Gewicht 1,023 besass, drehte im Decimeterrohr bei $20^{\circ}$ Natriumlicht $6,47^{\circ}$ nach links.

$$
[x]_{\mathrm{D}}^{200}=-71,94^{\circ} \text {. }
$$

II. $4,5192 \mathrm{~g}$ wässerige Lösung, welche $0,4061 \mathrm{~g}$ Sub$\operatorname{stanz}$, mithin $8,986 \%$ enthielt und das specifische Gewicht 1,024 besass, drehte im Decimeterrohr bei $20^{\circ}$ Natriumlicht $6,83^{\circ}$ nach links.

$$
[a]_{\mathrm{D}}^{200}=-74,22^{\circ} \text {. }
$$

III. $10,9570 \mathrm{~g}$ wässerige Lösung, welche $0,8094 \mathrm{~g} \mathrm{Sub}-$ stanz, mithin 7,39\% enthielt und das specifische Gewicht 1,02 besass, drehte im 2-Decimeterrohr bei $20^{\circ}$ Natriumlicht $11,67^{\circ}$ nach links.

$$
[x]_{\mathrm{D}}^{200}=-77,40^{\circ} \text {. }
$$

Der letzte Werth entspricht dem reinsten Präparat und ist deshalb am wahrscheinlichsten.

Von einem Präparat, welches in wässeriger Lösung $[a]_{D}^{200}=-72,64^{\circ}$ zeigte, wurde noch die Drehung in salzsaurer und in alkalischer Lösung bestimmt, um den Vergleich mit anderen Aminosäuren unter diesen Bedingungen zu ermöglichen.

Die Lösung in $20 \%$ iger Salzsäure, welche in $4,5904 \mathrm{~g}$ 0,3526 g Substanz, mithin 7,682\% enthielt und das specifische Gewicht 1,118 besass, drehte im Decimeterrohr bei $20^{\circ} \mathrm{Na}-$ triumlicht $4,00^{\circ}$ nach links.

$$
[a]_{\mathrm{D}}^{20^{0}}=-46,53^{\circ}
$$


Die alkalische Lösung (3 ccm. Normalkalilauge + Wasser), welche in 5,5106 g 0,3157 g Substanz, also 5,729\% enthielt und das specifische Gewicht 1,035 besass, drehte im Decimeterrohr bei $20^{\circ}$ Natriumlicht $4,95^{\circ}$ nach links.

$$
[c]_{\mathrm{D}}^{20^{\circ}}=-83,48^{\circ} \text {. }
$$

Die Zahlen sind selbstverständlich nicht ganz genau, genügen aber zur vorläufigen Orientirung.

Beim raschen Erhitzen im Capillarrohr schmolzen alle drei Präparate unter starker Gasentwicklung bei 203-206 ${ }^{\circ}$ $\left(206-209^{\circ}\right.$ corr.). Dabei tritt, ebenso wie beim Eindampfen des Kupfersalzes der charakteristische Geruch des Pyrrolidins auf.

Der endgültige Beweis, dass hier active $\alpha$-Pyrrolidincarbonsäure vorliegt, wurde überdies noch durch Verwandlung in die inactive Form geliefert. Die Racemisirung war nach 5 stündigem Erhitzen von $1 \mathrm{~g}$ Säure mit $2 \mathrm{~g}$ krystallisirtem Barythydrat und $4 \mathrm{~g}$ Wasser auf $140-145^{\circ}$ beendet. Nach der Fällung des Baryts mit Kohlensäure wurde das Kupfersalz dargestellt, welches jetzt in Alkohol völlig unlöslich war und aus Wasser krystallisirt die Zusammensetzung des a-pyrrolidincarbonsauren Kupfers hatte:

$0,2404 \mathrm{~g}$ lufttrockenes Salz verloren bei $110^{\circ} 0,0260 \mathrm{~g}$ an Gewicht und lieferten darnach $0,0594 \mathrm{~g} \mathrm{CuO}$.

$$
\begin{array}{ll}
\mathrm{C}_{10} \mathrm{H}_{16} \mathrm{O}_{4} \mathrm{~N}_{2} \mathrm{Cu}+2 \mathrm{H}_{2} \mathrm{O} . & \text { Berechnet: } 10,99 \% \mathrm{H}_{2} \mathrm{O} . \\
& \text { Gefunden: } 10,81 \% \\
\mathrm{C}_{10} \mathrm{H}_{16} \mathrm{O}_{4} \mathrm{~N}_{2} \mathrm{Cu} . & \text { Berechnet: } 21,81 \% \mathrm{Cu} . \\
& \text { Gefunden: } 22,13 \%
\end{array}
$$

Um die active Säure durch ein besser krystallisirtes Derivat $\mathrm{zu}$ charakterisiren, habe ich noch ihre Verbindung mit Phenylisocyanat bezw. deren Anhydrid dargestellt. $\mathrm{Zu}$ dem Zweck wurden 1,8 g der l-Pyrrolidincarbonsäure in $15 \mathrm{ccm}$. Normalnatronlauge gelöst und nach guter Abkühlung $2 \mathrm{~g}$ Phenylisocyanat in kleinen Portionen unter dauerndem kräftigen Schütteln zugefügt. Zum Schluss wird die Lösung mit etwas Tierkohle durchgeschüttelt, filtrirt und angesäuert, wobei die Phenylcyanatverbindung als harzige Masse ausfällt. Da sie wenig Neigung zum Krystallisiren hat, so fügt man noch so viel Salzsäure zu, dass die Lösung etwa $4 \%$ davon enthält, und engt auf dem Wasserbade ein, wobei an Stelle 
des ursprünglichen Oeles die Krystalle des Anhydrids treten. Diese werden nach dem Erkalten filtrirt und aus etwa $200 \mathrm{ccm}$. kochendem Wasser umkrystallisirt. Die Substanz ist sofort rein und die Ausbeute recht befriedigend.

In warmem Alkohol und Aceton ist sie erheblich leichter löslich als in Wasser, in Aether ist sie schwerer löslich. Sie krystallisirt aus diesen Flüssigkeiten meist in kleinen Prismen. Aus heissem Wasser, wovon etwa 110 Theile zur Lösung nöthig sind, fällt sie beim Erkalten in hübschen flachen Nadeln, welche bei $143^{\circ}$ (1440 corr.), mithin erheblich höher als die inactive Verbindung $\left(118^{\circ}\right)$ schmelzen.

Die im Vacuum über Schwefelsäure getrocknete Substanz gab folgende Zahlen:

$0,1952 \mathrm{~g}$ Substanz gaben $0,4770 \mathrm{~g} \mathrm{CO}_{2}$ und $0,0968 \mathrm{~g} \mathrm{H}_{2} \mathrm{O}$.

0,1960 » » $21,4 \mathrm{ccm} . \mathrm{N}\left(17^{\circ}, 769 \mathrm{~mm}\right.$.).

$\mathrm{C}_{12} \mathrm{H}_{12} \mathrm{O}_{2} \mathrm{~N}_{2}$. Berechnet: $66,67 \% \mathrm{C}, 5,57 \% \mathrm{H}, 12,96 \% \mathrm{~N}$. Gefunden: $66,64 \%$, 5,51\% $>12,83 \%$,

Bezüglich der Structur verweise ich auf das beim Racemkörper Gesagte, will aber hier der Bequemlichkeit halber die Formel anführen:<smiles>CCN(CC)C(=O)N1CCCC1C</smiles>

Wegen ihrer schönen Eigenschaften ist die Verbindung zur Erkennung der activen $\alpha$-Pyrrolidincarbonsäure recht geeignet.

Die Menge der Pyrrolidincarbonsäure, welche aus dem Casein entsteht, ist keineswegs gering, denn bei Anwendung von $500 \mathrm{~g}$ des Letzteren konnten allein aus der von $80-85^{\circ} \mathrm{g}$ siedenden Fraction der Ester $10 \mathrm{~g}$ active und $4 \mathrm{~g}$ racemische Säure isolirt werden. Aus der nächst niederen Fraction wurden noch weitere etwa $2 \mathrm{~g}$ gewonnen. Die Gesammtmenge der Säure betrug mithin 3,2\% des Caseins.

Wegen ihrer leichten Löslichkeit in $\mathrm{W}^{\top}$ asser und Alkohol ist sie allen früheren Beobachtern, welche sich mit der Spaltung des Caseins beschäftigt haben, entgangen. Auch bei anderen Proteinstoffen ist die Säure bisher nicht gefunden worden. Zwar gibt Schützenberger an, dass er aus Albumin 
durch Spaltung mit Barytwasser unter Druck ungesättigte Aminosäuren von der allgemeinen Formel $\mathrm{C}_{n} \mathrm{H}_{2 n-1} \mathrm{NO}_{2}$ die sogenannten Leuceine erhalten habe. Aber er hat keinen dieser Stoffe als chemisches Individuum charakterisirt. Er schliesst vielmehr auf ihre Existenz aus der Analyse von Präparaten, die nach ihrer Gewinnungsweise meiner Erfahrung gemäss nur Gemische gewesen sein können, und ich habe in keiner seiner Angaben auch nur eine Andeutung finden können, dass er die Pyrrolidincarbonsäure unter den Händen hatte.

Schwieriger ist die Frage zu entscheiden, ob die Säure ein primäres Spaltungsprodukt des Caseins ist; denn man kann sich auch wohl vorstellen, dass sie unter dem Einfluss der Salzsäure secundär aus einem 1,4 Derivat der Valeriansäure durch Ringschluss entstehe. Ich habe zunächst an das Arginin gedacht, welches durch Abspaltung der Guanidingruppe in Pyrrolidincarbonsäure übergehen könnte, und deshalb folgenden Versuch ausgeführt:

$1 \mathrm{~g}$ Argininnitrat, welches mir von Herrn E. Schulze freundlichst zur Verfügung gestellt war, wurde zuerst mit $1^{1 / 2}$ Molekülen Schwefelsäure in verdünnter wässeriger Lösung versetzt und mehrmals unter vermindertem Druck abdestillirt, um die Salpetersäure zu entfernen. Dann wurde das Sulfat mit $20 \mathrm{ccm}$. rauchender Salzsäure erst 6 Stunden am Rückflusskühler gekocht und schliesslich genau in derselben Weise wie die Caseinlösung auf die Ester der Aminosäuren verarbeitet. Da unter diesen Bedingungen gar kein in Aether löslicher Ester erhalten wurde, so war offenbar keine Pyrrolidincarbonsäure gebildet worden.

Ebensowenig gelang es, das Ornithin in Pyrrolidincarbonsäure zu verwandeln.

Für den Versuch diente synthetische Ornithursäure. 1,5 g wurden mit $30 \mathrm{ccm}$. Salzsäure (25\%) 10 Stunden am Rückflusskühler gekocht, nach dem Abkühlen von der Benzoesäure abfiltrirt, noch 5 Stunden gekocht, verdampft und der Rückstand zur Entfernung der Benzoesäure mit Aether behandelt. Der zurückbleibende Sirup wurde dann in der üblichen Weise verestert. Es resultirte dabei in der That ein in Aether lös- 
liches Produkt, welches nach dem Verjagen des Aethers durch Kochen mit Wasser verseift und auf Kupfersalz verarbeitet wurde. Beim Eindampfen der grünen Kupfersalzlösung trat allerdings ein stark basischer Geruch auf, welcher demjenigen des Pyrrolidins ähnlich war, aber ebenso gut von Tetramethylendiamin herrühren konnte. Das Kupfersalz war indessen nicht allein durch die Farbe, sondern auch durch die ausserordentlich leichte Löslichkeit in kaltem Wasser von dem Salz der racemischen Pyrrolidincarbonsäure verschieden. Ich glaube deshalb annehmen $\mathrm{zu}$ dürfen, dass Pyrrolidincarbonsäure in irgendwie erheblicher Menge bei dem Versuch nicht entstanden war.

Um die Frage auch noch von einer anderen Seite her in Angriff zu nehmen, habe ich in Gemeinschaft mit Herrn Dr. P. A. Levene einige Versuche über die Spaltung des Caseins mit Trypsin angestellt, bei welchen die Salzsäure nur in alkoholischer Lösung zur Veresterung der Aminosäuren in Anwendung kam. Da auch hier die Entstehung von Pyrrolidincarbonsäure beobachet wurde, so halte ich es nach allen bisher vorliegenden Erfahrungen für sehr wahrscheinlich, dass sie ein primäres Spaltungsprodukt ist.

Da hierdurch die Verbindung, welche zum Unterschied von allen bisher aus Eiweisskörpern durch Hydrolyse erhaltenen Aminosäuren einen stickstoffhaltigen Ring enthält, ein erhöhtes Interesse gewinnt, so habe ich die Absicht, noch eine Reihe anderer Proteinstoffe in der gleichen Art zu prüfen. Nach Versuchen des Herrn Dr. Georg Franz kann ich schon jetzt erwähnen, dass das käufliche Blutfibrin bei der Spaltung mit Salzsäure gleichfalls Pyrrolidincarbonsäure liefert.

Die Fraction $85-110^{\circ}$, deren Menge relativ klein war, enthielt noch etwas Leucin neben anderen Produkten, die nicht näher untersucht sind.

\section{Fraction $110-120^{\circ}$.}

Da dieselbe erhebliche Mengen Asparaginsäureester enthält, welcher beim Kochen mit Wasser eine complexe Zersetzung erfährt, so wurde zur Verseifung mit überschüssigem, 
etwa 20\% igem Barytwasser auf dem Wasserbade erhitzt. Nach kurzer Zeit begann die Abscheidung eines schwach gelb gefärbten krystallinischen Barytsalzes. Je nach dem Ueberschuss von Baryt ist die Verseifung in 1-2 Stunden beendet. Man lässt dann erkalten und filtrirt das abgeschiedene Salz. Dasselbe ist asparaginsaurer Baryt. Die daraus in bekannter Weise isolirte Asparaginsäure gab folgende Zahlen:

0,1987 g Substanz lieferten 0,2617 $\mathrm{g} \mathrm{CO}_{2}$ und 0,0973 $\mathrm{g} \mathrm{H}_{2} \mathrm{O}$.

$\mathrm{C}_{4} \mathrm{H}_{7} \mathrm{O}_{4} \mathrm{~N}$. Berechnet: $36,09 \% \mathrm{C}, 5,26 \% \mathrm{H}$.

Gefunden: $35,92 \% \gg 5,40 \%$ »

Es verdient hervorgehoben $\mathrm{zu}$ werden, dass die Säure zum grösseren Theile racemisirt war. Die Lösung in Salzsäure $(10 \%)$, welche $8,175 \%$ Substanz enthielt und das specifische Gewicht 1,05 besass, drehte im 2-Decimeterrohr bei $20^{\circ}$ Natriumlicht $1,31^{\circ}$ nach rechts. Also $[\alpha]_{D}^{200}=+7,63$, während die reine active Asparaginsäure unter den gleichen Bedingungen $+26,5^{0}$ gab. $^{1}$ ) Die Substanz enthielt also nur 29\% activer Säure. Dadurch erklärt sich auch die leichte Abscheidung des Barytsalzes, denn das neutrale Salz der activen Säure ist in heissem Wasser sehr leicht löslich und kommt unter den Bedingungen des obigen Versuches kaum zur Abscheidung.

Die vom Barytsalz abfiltrirte Mutterlauge wurde mit Schwefelsäure vom Baryt befreit und verdampft. Sie enthielt ausser Asparaginsäure noch leicht lösliche Aminosäuren, über deren Natur ich vorläufig nichts sagen kann.

Die Fraction $120-130^{\circ}$ enthält viel Asparaginsäure, wahrscheinlich auch kleine Mengen Glutaminsäure, und ausserdem in Wasser sehr leicht lösliche Stoffe, die beim Eindampfen einen eigenthümlichen, an Fleischextract erinnernden Geruch geben und wahrscheinlich serinähnliche Stoffe sind. Ich beabsichtige, dieselben noch zu untersuchen. Ferner ist in dieser Fraction der Ester des Phenylalanins vorhanden.

Um ihn von den leicht löslichen Estern der Glutamin-

1) E. Fischer, Ber. d. deutsch. chem. Ges., 32, 2463, 1899. 
und Asparaginsäure zu trennen, wird das Gemisch mit der 7 fachen Menge kaltem Wasser geschüttelt, das ausgeschiedene Oel am besten durch Filtration auf einem nassen Papierfilter abgetrennt und noch 2 Mal mit der 3fachen Menge Wasser gewaschen. Zur Umwandlung in Phenylalanin wird der rohe Ester mit der doppelten Menge Barythydrat und der 10fachen Menge Wasser unter häufigem Umschütteln mehrere Stunden auf dem Wasserbade erwärmt. Dabei bleibt ein Theil des Oels ungelöst, das sind Produkte unbekannter Zusammensetzung. Aus der wässerigen Lösung wird der Baryt quantitativ mit Schwefelsäure gefällt und beim Verdampfen bleibt dann das Phenylalanin als fast farblose, meist aus Blättchen bestehende Krystallmasse zurück. Seine völlige Reinigung bietet besondere Schwierigkeiten. Ein mehrmals aus Wasser umkrystallisirtes Präparat gab folgende Zahlen, welche noch auf eine merkliche Verunreinigung hindeuten:

$0,1811 \mathrm{~g}$ Sbst. lieferten $0,4300 \mathrm{~g} \mathrm{CO}_{2}$ und $0,1087 \mathrm{~g} \mathrm{H}_{2} \mathrm{O}$.

0,1646 » >11,9 ccm. $\mathrm{N}\left(15^{\circ}, 761 \mathrm{~mm}\right)$.

$\mathrm{C}_{9} \mathrm{H}_{11} \mathrm{O}_{2} \mathrm{~N}$. Berechnet: $65,42 \% \mathrm{C}, 6,66 \% \mathrm{H}, 8,48 \% \mathrm{~N}$. Gefunden: $64,76 \%$ \6,69\% $8,46 \%$,

Zudem ist das Präparat ein Gemisch von racemischem und activem Phenylalanin, deren Mengenverhältniss selbstverständlich bei verschiedenen Krystallisationen variirt. Die active Form ist die l-Verbindung, mithin die gleiche, welche E. Schulze zuerst in verschiedenen pflanzlichen Proteinstoffen gefunden hat. Dementsprechend dreht die wässerige Lösung des Gemisches nach links, aber die Stärke der Drehung schwankt. Ein ähnliches Resultat gab die Untersuchung der Phenylisocyanatverbindung. Auch hier waren Schmelzpunkt und Drehungsvermögen nicht constant. Für ein Präparat, welches bei $164^{\circ}$ (166 corr.) schmolz, waren die Zahlen folgende:

4,4599 g Lösung, welche $1,4 \mathrm{ccm}$. Normalkalilauge und $0,3647 \mathrm{~g}$ Substanz, mithin $8,18 \%$ enthielt und das specifische Gewicht 1,03 besass, drehte im Decimeterrohr bei $20^{\circ}$ Natriumlicht $4,16^{\circ}$ nach rechts. $[\alpha]_{\mathrm{D}}^{20^{\circ}}=+49,35^{\circ}$, wäh- 
rend das Drehungsvermögen des reinen Phenylisocyanat-lPhenylalanins $+61,3$ sein müsste. ${ }^{1}$ )

Ein anderes Präparat des Phenylisocyanatkörpers wurde für die Analyse verwendet:

$0,2006 \mathrm{~g}$ Substanz lieferten $0,4974 \mathrm{~g} \mathrm{CO}_{2}$ und $0,1034 \mathrm{~g} \mathrm{H}_{9} \mathrm{O}$.

$\mathrm{C}_{16} \mathrm{H}_{18} \mathrm{O}_{3} \mathrm{~N}_{2}$. Berechnet: $67,60 \% \mathrm{C}, 5,63 \% \mathrm{H}$.

Gefunden: $67,62 \%$, 5,73\% 》

Da aber immer noch Differenzen im Schmelzpunkt mit den genau untersuchten synthetischen Produkten vorhanden waren, so wurde schliesslich das Phenylalanin aus Casein durch 48stündiges Erhitzen mit der 3fachen Menge Barythydrat und der 20fachen Menge Wasser auf 155-160 völlig racemisirt. Nach Fällung des Baryts mit Schwefelsäure und Eindampfen der Lösung schied sich jetzt das racemische Phenylalanin in glänzenden Blättchen $\mathrm{ab}$, welche bekanntlich viel schwerer löslich sind als die active Form. Nach einmaligem Umkrystallisiren aus Wasser enthielt die bei $100^{\circ}$ getrocknete Substanz noch etwas zu wenig Kohlenstoff:

$0,1781 \mathrm{~g}$ Substanz gaben 0,4242 $\mathrm{g} \mathrm{CO}_{2}$ und $0,1087 \mathrm{~g} \mathrm{H}_{2} \mathrm{O}$.

$\mathrm{C}_{9} \mathrm{H}_{11} \mathrm{O}_{2} \mathrm{~N}$. Berechnet: $65,42 \% \mathrm{C}, 6,66 \% \mathrm{H}$.

Gefunden: $64,96 \%$, $6,78 \%$,

Die aus diesem Präparat hergestellte Phenylisocyanatverbindung zeigte dann in Krystallform und Schmelzpunkt (gef. 180-1810 corr.) völlige Uebereinstimmung mit dem synthetischen Produkt. Dasselbe traf auch noch zu für das durch Kochen mit Salzsäure entstehende Anhydrid. Dadurch werden die letzten Zweifel an der Entstehung von Phenylanalin aus Casein beseitigt.

Die Menge des Phenylalanins ist gering. Bei Anwendung von $500 \mathrm{~g}$ Casein wurden aus der Fraction 130-160 6 g Rohprodukt gewonnen. Da etwas Ester beim Waschen mit Wasser

1) Die Bestimmung wurde bisher nur mit der d-Verbindung ausgeführt (E. Fischer u. A. Mouneyrat, Ber. d. deutsch. chem. Gesellsch., 33, 2386). Leider ist hier durch ein Versehen die Drehungsrichtung falsch angegeben. Ich habe den Versuch nochmals wiederholt. Das Phenylisocyanat-d-Phenylalanin dreht in alkalischer Lösung nach links. Es ist also hier $\mathrm{zu}$ setzen $[\alpha]_{\mathrm{D}}^{20^{\circ}}=-61,3^{\circ}$. 
verloren geht und da eine andere, allerdings kleinere Menge sich in der niedrigeren Fraction findet, da ferner die Estermethode selbst beim reinen Präparat ungefähr 55\% Verluste bringt, so darf man annehmen, dass die Menge des Phenylalanins nicht weniger als $21 / 2 \%$ des Caseins beträgt. Trotzdem würde seine Isolirung ohne die Estermethode wohl recht schwierig sein, da für die Trennung von dem Leucin kein brauchbares Verfahren bekannt ist. Thatsächlich ist E. Schulze, wohl der beste Kenner des Phenylalanins, welcher es zuerst unter den Spaltungsprodukten der pflanzlichen Proteine fand, bei Anwendung von $1 \mathrm{~kg}$ Casein nicht zum Ziele gelangt, obschon er bei der Oxydation der rohen Aminosäuren mit Bichromat und Schwefelsäure Benzoesäure erhielt und dadurch auf die Vermuthung kam, dass Phenylalanin vorhanden sein könnte. ${ }^{1}$ )

Der Bildung der Benzoesäure soll nach den Angaben von Schulze und Barbieriª) der Geruch nach Benzaldehyd voraufgehen. Ich halte es auch für sehr wahrscheinlich, dass in dem späteren Stadium der Oxydation Benzaldehyd auftritt, aber das Produkt, welches sich zuerst bildet, hat den charakteristischen Geruch des Phenylacetaldehyds, und es lassen sich mit Hülfe desselben noch recht kleine Mengen Phenylalanin nachweisen. So genügt es, $0,02 \mathrm{~g}$ in $2-3 \mathrm{ccm}$. 25\% \% iger Schwefelsäure zu lösen, dann ein Paar Körnchen Bichromat hinzuzufügen und zu kochen, um sehr deutlich den Geruch des Aldehyds zu erhalten. ${ }^{3}$ )

\section{Zusammenfassung der Resultate:}

1. Die Trennung der Monoaminosäuren durch fractionirte Destillation ihrer Ester ist den bisher gebräuchlichen Methoden

1) Diese Zeitschr., Bd. IX, S. 120, 1885.

2) Journ. f. prakt. Chem., 27, 345, 1883.

3) Etwas beständiger sind die fetten Aminosäuren gegen oxydirende Agentien. So wird das Alanin in verdünnter schwefelsaurer Lösung von Chromsäure nur langsam angegriffen. Löst man aber dasselbe in $25 \%$ iger Schwefelsäure, fügt einige Körnchen Kaliumpermanganat hinzu und kocht, so tritt nach kurzer Zeit auch der Geruch nach Acetaldehyd auf. Die Bildung von Aldehyden bei der Oxydation von Aminosäuren erfolgt offenbar unter Abspaltung von Kohlensäure und Ammoniak. 
zur Isolirung und Erkennung dieser Säuren sowohl an Leistungsfähigkeit wie an Schnelligkeit der Ausführung weit überlegen. Eine Ausnahme besteht nur für das Tyrosin, welches viel leichter durch direkte Krystallisation gewonnen wird;

2. unter den Spaltungsprodukten des käuflichen Caseins durch Salzsäure sind ausser den schon beobachteten Aminosäuren sicher nachgewiesen: Phenylalanin und eine Aminovaleriansäure von unbekannter Structur. Sehr wahrscheinlich ist auch die Anwesenheit von Glycocoll gemacht;

3. bei der Hydrolyse des Caseins durch Salzsäure entstehen erhebliche Mengen von $\alpha$-Pyrrolidincarbonsäure, die bisher unter den Spaltungsprodukten der Proteinstoffe noch nicht gefunden war. Ein Theil der Säure ist in der bisher unbekannten activen l-Form isolirt worden, der andere Theil war racemisirt. Die Säure scheint ein primäres Spaltungsprodukt des Caseins zu sein und entsteht auch aus anderen Proteinstoffen, z. B. dem Blutfibrin;

4. die mit der Estermethode isolirten Aminosäuren sind theilweise racemisirt, speciell wurde dies nachgewiesen für Leucin, Phenylalanin und Asparaginsäure. Für die Isolirung der einzelnen Säuren durch Krystallisation ist es deshalb rathsam, die ganze Menge durch Erhitzen mit Barytwasser unter Druck vorher zu racemisiren;

5. für die Identificirung der Aminosäuren leistet die Untersuchung der Phenylisocyanatverbindung und ihres Anhydrids manchmal sehr gute Dienste;

6. die schon von E. Schulze bei Gemischen beohachtete Veränderung in der Löslichkeit der aminosauren Kupfersalze hat sich an neuen auffallenden Beispielen wieder gezeigt. So wird das Kupfersalz der Aminovaleriansäure bei Gegenwart von l-Pyrrolidincarbonsäure in absolutem Alkohol löslich. Ferner bildet Leucinkupfer mit dem aminovaleriansauren Kupfer in Wasser leicht lösliche grosse Krystalle, welche die Zusammensetzung einer molekularen Verbindung zeigen;

7. Der Ester des Phenylalanins lässt sich von den Estern der Glutamin- und Asparaginsäure, welche ungefähr den gleichen Siedepunkt haben, durch Behandlung mit Wasser leicht trennen. 
176 Emil Fischer, Ueber die Hydrolyse des Caseins, etc.

Beim Kochen von Phenylalanin mit Schwefelsäure undl Kaliumbichromat tritt der Geruch von Phenylacetaldehyd auf,; und diese Reaction bildet eine ebenso scharfe wie bequemee Methode zur Auffindung des Phenylalanins.

Die vorstehenden Versuche waren zum Theil aussergewöhnlich mühsam und langwierig. Um so mehr fühle ich mich meinem Assistenten, Herrn Dr. Otto Wolfes, für die dabei geleistete Hülfe zu grossem Dank verpflichtet. 